\title{
Factors affecting pre- and post-stenting computed tomography perfusion in patients with middle cerebral artery stenosis
}

\author{
HUI QU, JINLI LI, XINGQUAN ZHAO and KEHUI DONG
}

Department of Neurology, Beijing Tiantan Hospital affiliated to Capital Medical University, Beijing 100050, P.R. China

Received September 6, 2012; Accepted October 22, 2012

DOI: $10.3892 /$ etm.2012.805

\begin{abstract}
The aim of this study was to investigate the factors affecting pre- and post-stenting head computed tomography perfusion (CTP) in patients with middle cerebral artery stenosis. A total of 25 patients with severe middle cerebral artery stenosis were enrolled. CTP was performed prior to and following stenting. Scores were allocated to the time-to-peak (TTP) parameter of CTP using the Alberta stroke program early computed tomography scoring (ASPECTS) scale. The factors possibly affecting pre- and post-stenting CTP were analyzed. All the patients exhibited markedly prolonged TTP on the affected side prior to stenting, compared with the healthy side. Following surgery, the TTP was improved in all patients. The preoperative ASPECTS score was negatively correlated with the degree of middle cerebral artery stenosis with a correlation coefficient of -5.78 . The preoperative vascular stenosis rate was positively correlated with the improvement degree of the ASPECTS score with a correlation coefficient of 1.137 $(\mathrm{P}=0.001)$. TTP is a sensitive parameter for evaluating the effect of stenting on middle cerebral artery stenosis. TTP prior to and following stenting may be quantitatively assessed using the ASPECTS scale. Patients with serious stenosis and/or good collateral circulation are able to benefit more from stenting.
\end{abstract}

\section{Introduction}

The head computed tomography perfusion (CTP) technique is an important method for cerebral blood flow assessment (1-4). This technique visually represents the blood flow perfusion in various parts of the brain tissue. In the application of CTP, time to peak (TTP) is the most sensitive and specific index for brain ischemia (5). Stenting is a technique which has been applied in cerebrovascular disease treatment, particularly for ischemic cerebrovascular diseases (6-10). The effect of stenting on isch-

Correspondence to: Professor Hui Qu, Department of Neurology, Beijing Tiantan Hospital affiliated to Capital Medical University, Tiantan xili 6, Dongcheng, Beijing 100050, P.R. China

E-mail: qhkhcn@163.com

Key words: computed tomography perfusion, time to peak, stent, Alberta stroke program early computed tomography scoring emic cerebrovascular disease may be evaluated visually using the CTP technique $(11,12)$.

In the present study, the Alberta stroke program early computed tomography scoring (ASPECTS) scale was used to quantitatively analyze CTP in 25 patients with ischemic cerebrovascular disease caused by middle cerebral artery solitary stenosis prior to and following stenting. The factors affecting CTP prior to and following stenting were further analyzed $(13,14)$.

\section{Patients and methods}

Clinical data. A total of 25 patients with cerebrovascular disease caused by unilateral middle cerebral artery solitary stenosis that received treatment at Tiantan Hospital (Beijing, China) between January 1, 2004 and March 1, 2007 were enrolled. All patients were subjected to percutaneous stent implantation (stenting). Head CTP imaging was performed within 7 days before and after stenting. Of the 25 patients, 12 had transient ischemic attack (TIA) and 13 had cerebral infarction (CI). The patient's ages ranged between 34 and 71 years with an average of $49.4 \pm 8.4$ years. Of the patients, 19 were male and 6 were female. The degree of stenosis ranged between 60 and $99 \%$ with an average of $80.1 \pm 11.4 \%$. This study was conducted with approval from the Ethics Committee of Capital Medical University. Written informed consent was obtained from all participants.

Pre-stenting evaluation. Preoperative evaluation included angiographic classification of the cerebral arterial stenosis based on Mori's method $(15,16)$, clinical typing of the cerebral arterial stenosis (6) and classification based on whether apparent cerebral blood flow collateral circulation existed (17).

CTP examination. CT scanning was performed using a GE Lightspeed spiral CT system. The scanning matrix was $512 \times 512$ and the exposure conditions were $120 \mathrm{kV}$ and $100 \mathrm{~mA}$. Successive scanning of the layers of interest began when a high pressure injector was started for rapid intravenous injection of the contrast medium. The slice thickness was $10 \mathrm{~mm}$ and the scanning lasted $60 \mathrm{sec}$ at a 1 slice/sec scanning rate (60 slices in total). Iohexol $(300 \mathrm{mg} / \mathrm{ml})$ was used as the contrast medium, with a volume of $40 \mathrm{ml}$ at an $8 \mathrm{ml} / \mathrm{sec}$ flow rate. The layers of interest included the basal and coronal radiation layers. The regional cerebral blood flow (rCBF), regional 


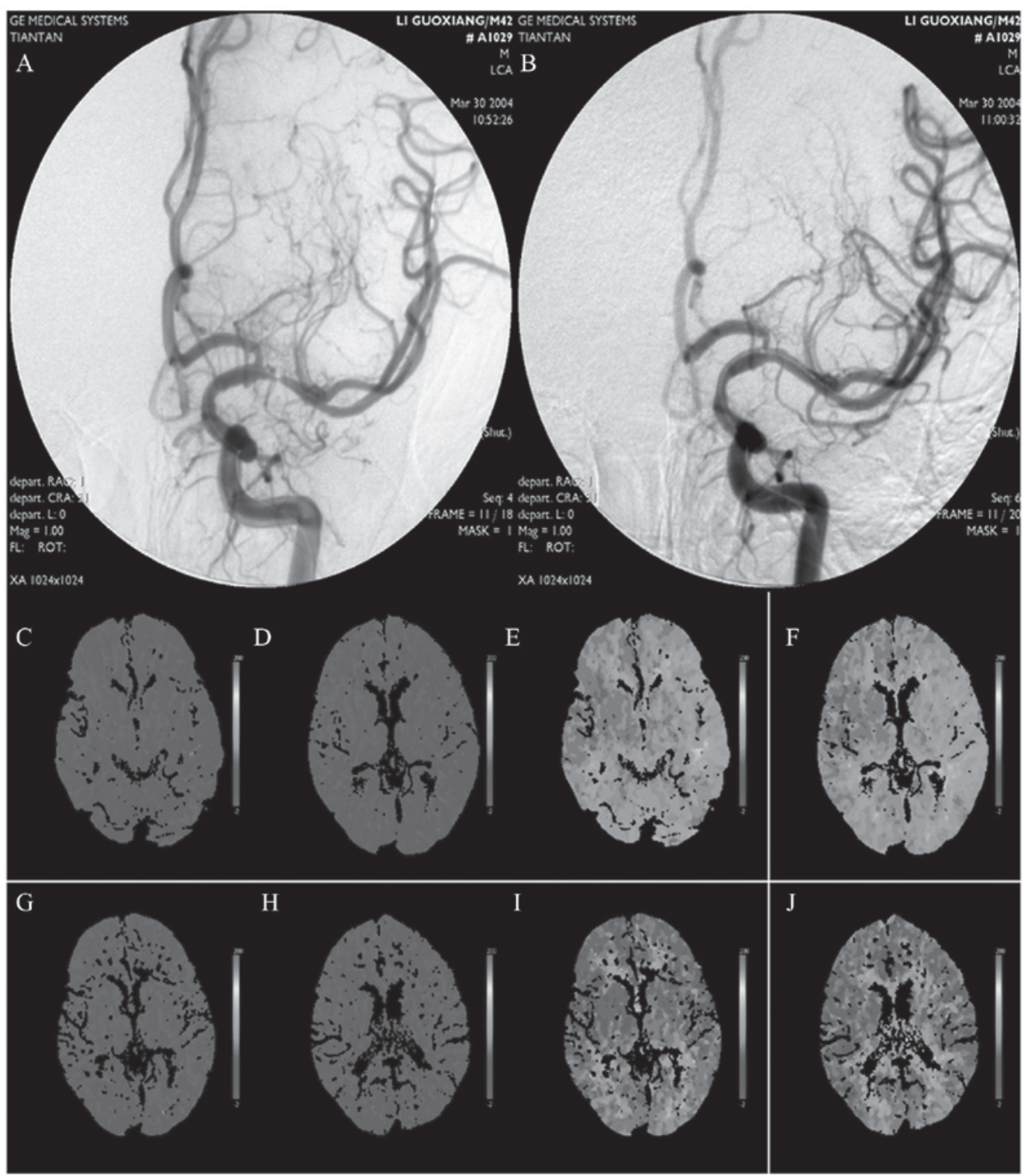

Figure 1. DSA and CT images of patients with middle cerebral artery stenosis. (A) Preoperative stenosis rate of 99\% at the M1 segment of the LMCA by DSA; (B) no notable postoperative residual LMCA stenosis in the same patient by DSA; (C-F) preoperative prolonged MTT and TTP at the left frontal lobe, left temporal and bilateral occipital lobes, respectively, by cranial perfusion CT; and (G-J) postoperative, essentially normal MTT and TTP at the left frontal and temporal lobes but high MTT and TTP at the bilateral occipital lobes. LMCA, left middle cerebral artery; DSA, digital subtraction angiography; MTT, mean transit time; TTP, time-to-peak.

cerebral blood volume (rCBV), mean transit time (MTT) and TTP parameters were calculated using professional software. Color maps were obtained.

Pre- and post-stenting CTP scoring criteria. The CT parameter color maps prior to and following stenting were compared and the head CTP TTP prior to and following stenting was scored using the ASPECTS scale $(13,14)$. The highest possible mark of the scale is 10 points. Higher scores indicates greater cerebral perfusion. The TTP improvement degree was then calculated based on the following formula: TTP improvement degree $=($ post-stenting ASPECTS score - pre-stenting ASPECTS score)/10x100(\%).

Statistical analysis. The $\chi^{2}$ test was used to compare categorical data, and t-tests or paired t-tests were used to compare continuous data. Correlation analysis and linear regression were performed to calculate the correlation coefficient and regression equation. All data were analyzed using SPSS 11.5 statistical software.

\section{Results}

The CTP color maps revealed that the TTP values in all the 25 patients were significantly prolonged prior to surgery, while following surgery, all these values improved by various degrees. The ASPECTS score (mean \pm SD) prior to surgery was $2.32 \pm 1.31$, whereas that following surgery was $8.28 \pm 1.65$, indicating a significant difference $(\mathrm{P}<0.01)$. The preoperative ASPECTS score was negatively correlated with the degree of middle cerebral artery solitary stenosis with a correlation coefficient of -5.78 (Fig. 1). 
Table I. T-test analysis of the factors affecting pre- and post-operative CTP.

\begin{tabular}{|c|c|c|c|c|}
\hline Factor & Pre-stenting ASPECTS & Post-stenting ASPECTS & Improvement degree (\%) & P-value \\
\hline Gender & & & & 0.121 \\
\hline Male & $2.26 \pm 1.33$ & $8.58 \pm 1.50$ & $63.16 \pm 17.34$ & \\
\hline Female & $2.50 \pm 1.38$ & $7.33 \pm 1.86$ & $48.33 \pm 26.40$ & \\
\hline High blood pressure & & & & 0.782 \\
\hline Yes & $2.36 \pm 1.03$ & $8.45 \pm 1.75$ & $60.91 \pm 20.72$ & \\
\hline No & $2.29 \pm 1.54$ & $8.14 \pm 1.61$ & $58.57 \pm 20.70$ & \\
\hline Diabetes & & & & 0.462 \\
\hline Yes & $1.50 \pm 0.71$ & $8.50 \pm 2.12$ & $70.00 \pm 14.14$ & \\
\hline No & $2.39 \pm 1.34$ & $8.26 \pm 1.66$ & $58.70 \pm 20.74$ & \\
\hline Coronary heart disease ${ }^{a}$ & & & & 0.315 \\
\hline Yes & 2.00 & 10.00 & 80.00 & \\
\hline No & $2.33 \pm 1.34$ & $8.21 \pm 1.64$ & $58.75 \pm 20.28$ & \\
\hline Smoking & & & & 0.426 \\
\hline Yes & $2.17 \pm 1.10$ & $8.33 \pm 1.50$ & $61.67 \pm 18.23$ & \\
\hline No & $2.71 \pm 1.80$ & $8.14 \pm 2.12$ & $54.29 \pm 25.73$ & \\
\hline Drinking & & & & 0.384 \\
\hline Yes & $2.50 \pm 1.17$ & $8.08 \pm 1.51$ & $55.83 \pm 18.32$ & \\
\hline No & $2.15 \pm 1.46$ & $8.46 \pm 1.81$ & $63.08 \pm 22.13$ & \\
\hline Dyslipidemia & & & & 0.190 \\
\hline Yes & $2.00 \pm 0.93$ & $8.40 \pm 1.80$ & $64.00 \pm 19.57$ & \\
\hline No & $2.80 \pm 1.69$ & $8.10 \pm 1.45$ & $53.00 \pm 20.58$ & \\
\hline Hyperhomocysteinemia & & & & 0.484 \\
\hline Yes & $2.43 \pm 1.27$ & $8.86 \pm 1.21$ & $64.29 \pm 19.02$ & \\
\hline No & $2.28 \pm 1.36$ & $8.06 \pm 1.76$ & $57.78 \pm 21.02$ & \\
\hline Discharge diagnosis & & & & 0.286 \\
\hline TIA & $2.83 \pm 1.47$ & $8.33 \pm 1.61$ & $55.00 \pm 21.95$ & \\
\hline $\mathrm{CI}$ & $1.84 \pm 0.99$ & $8.23 \pm 1.74$ & $63.85 \pm 18.50$ & \\
\hline \multicolumn{5}{|l|}{ Collateral circulation } \\
\hline Yes & $2.91 \pm 1.38$ & $7.91 \pm 2.02$ & $50.00 \pm 21.91$ & 0.033 \\
\hline No & $1.86 \pm 1.10$ & $8.57 \pm 1.28$ & $67.14 \pm 15.90$ & \\
\hline
\end{tabular}

CTP, computed tomography perfusion; ASPECTS, Alberta stroke program early computed tomography scoring; TIA, transient ischemic attack; CI, cerebral infarction. ${ }^{a}$ One patient with coronary heart disease was analyzed.

Table II. Correlation analysis of the factors possibly affecting pre- and post-stenting CTP.

\begin{tabular}{lc}
\hline Factor & P-value \\
\hline Age & 0.839 \\
Preoperative vascular stenosis rate & 0.001 \\
Postoperative residual stenosis rate & 0.923 \\
Pre-stenting NIHHS score & 0.668 \\
Post-stenting NIHHS score & 0.596
\end{tabular}

CTP, computed tomography perfusion; NIHHS, National Institutes of Health stroke scale.

Furthermore, the correlation factors possibly affecting pre- and post-stenting CTP improvement were analyzed and compared between the subgroups. These factors included gender, discharge diagnosis of manifestations and vessels (including CI and TIA), collateral circulation, histories of high blood pressure, diabetes, coronary heart disease and smoking, as well as dyslipidemia and hyperhomocysteinemia. The comparisons revealed that only the collateral circulation subgroups exhibited a significant difference $(\mathrm{P}=0.033)$. The results are shown in Table I. Correlations of age, the preoperative vascular stenosis rate, postoperative residual stenosis rate and pre- and post-operative scores based on the National Institutes of Health stroke scale (NIHHS) with CTP improvement were analyzed. The results revealed that only the preoperative vascular stenosis rate was positively correlated with the CTP ASPECTS score improvement with a correlation coefficient of 1.137 ( $\mathrm{P}=0.01$; Table II).

\section{Discussion}

Cerebral arterial stenosis is a significant pathological mechanism leading to ischemic cerebrovascular disease. Theoretically, the 
removal of stenosis and improvement of the cerebral blood flow is likely to decrease the incidence of CI. Percutaneous endovascular stenting has been demonstrated to be an effective treatment method for intracranial cerebral arterial stenosis (6-8), although evaluations of the effectiveness of stenting are often based on long-term stroke and preoperative event incidence rates $(6,7)$. CTP is highly sensitive to the improvement effect of stenting on cerebral perfusion. CTP color maps visually represent the cerebral perfusion improvement following stenting $(12,18)$ but are not contributory to scientific research statistics. The ASPECTS scale was an early tool for evaluating the effect of thrombolytic therapy on CI $(13,14)$. Since the radiological layers involved in the ASPECTS scale are the same as the CT scanning layers involved in the CTP technique, the scale may also be used for evaluating middle cerebral arterial blood supply.

All 25 patients in the present study had middle cerebral artery solitary stenosis. The CTP color maps revealed that their TTP was prolonged prior to stenting, whereas following stenting, the values were improved significantly. These findings were consistent with those reported previously $(11,12)$. The ASPECTS score also revealed a significant difference in the cerebral perfusion prior to and following stenting $(\mathrm{P}<0.01)$, which indicated a perfusion improvement following stenting. This result suggests that the evaluation of CTP improvement using the ASPECTS scale is feasible. However, although the ASPECTS score bore a negative correlation with the degree of severity of middle cerebral stenosis prior to stenting, a correlation was not observed between the ASPECT score and residual stenosis following stenting. When the middle cerebral stenosis was between $60 \%$ and $99 \%$, the more serious stenosis led to a lower ASPECT score, whereas when residual stenosis fell below $30 \%$, the ASPECT score did not appear to vary according to the severity of stenosis. This suggests that the ASPECTS scale has statistical significance only within certain degrees of stenosis.

Furthermore, the correlation factors which may affect CTP prior to and following stenting were also analyzed in the present study. The studied factors included age, gender, histories of high blood pressure, smoking, drinking, diabetes and coronary heart disease, as well as hyperlipidemia, hyperhomocysteinemia, postoperative residual stenosis rate, NIHHS score prior to and following surgery and discharge diagnosis (TIA and CI). The analysis revealed that the preoperative vascular stenosis rate and collateral circulation were the only factors correlated with the degree of CTP improvement. This result suggests that the non-correlated factors are negligible in surgical patient selection and prognosis assessment. The preoperative vascular stenosis rate was markedly correlated with the degree of postoperative CTP improvement. A more serious degree of stenosis indicated a more marked improvement effect of stenting for cerebral perfusion and cerebral ischemia. In addition, whether there was good collateral circulation in the blood supply region prior to stenting also greatly affected the degree of postoperative CTP improvement. Patients without good collateral circulation exhibited greater degrees of CTP improvement. This suggests that patients without good collateral circulation benefit more from stenting, which is in agreement with the reported literature (7).

Based on the findings of the present study, the degree of vascular stenosis and whether good collateral circulation exists should be considered first when stenting is selected for patients with intracranial arteriostenosis, in order to achieve satisfactory cerebral perfusion improvement. The considerations of age, gender, drinking, high blood pressure, diabetes, coronary heart disease, lipid disorders and hyperhomocysteinemia should then be considered. However, the present study had a significant limitation. In order to remove the effects of external factors, all the recruited patients were those with single cerebral artery disease. The patients received CTP detection prior to and following stenting. Due to the strict selection criteria, the sample size in the present study was small with only 25 subjects enrolled.

\section{References}

1. Gasparotti R, Grassi M, Mardighian D, et al: Perfusion CT in patients with acute ischemic stroke treated with intra-arterial thrombolysis: predictive value of infarct core size on clinical outcome. AJNR 30: 722-727, 2009.

2. d'Esterre CD, Aviv RI and Lee TY: The evolution of the cerebral blood volume abnormality in patients with ischemic stroke: a CT perfusion study. Acta Radiol 53: 461-467, 2012.

3. König M: Brain perfusion CT in acute stroke: current status. Eur J Radiol 45 (Suppl 1): S11-S22, 2003.

4. Galvez M, York GE 2nd and Eastwood JD: CT perfusion parameter values in regions of diffusion abnormalities. AJNR Am J Neuroradiol 25: 1205-1210, 2004.

5. Smith WS, Roberts HC, Chuang NA, et al: Safety and feasibility of a CT protocol for acute stroke: combined CT, CT angiography, and CT perfusion imaging in 53 consecutive patients. AJNR Am J Neuroradiol 24: 688-690, 2003.

6. Jiang WJ, Wang YJ, Du B, et al: Stenting of symptomatic M1 stenosis of middle cerebral artery: an initial experience of 40 patients. Stroke 35: 1375-1380, 2004.

7. Chimowitz MI, Lynn MJ, Derdeyn CP, et al: Stenting versus aggressive medical therapy for intracranial arterial stenosis. N Engl J Med 365: 993-1003, 2011.

8. Gröschel K, Schnaudigel S, Pilgram SM, Wasser K and Kastrup A: A systematic review on outcome after stenting for intracranial atherosclerosis. Stroke 40: e340-e347, 2009.

9. SSYLVIA Study Investigators. Stenting of Symptomatic Atherosclerotic Lesions in the Vertebral or Intracranial Arteries (SSYLVIA): study results. Stroke 35: 1388-1392, 2004.

10. Suh DC, Kim JK, Choi JW, et al: Intracranial stenting of severe symptomatic intracranial stenosis: results of 100 consecutive patients. AJNR Am J Neuroradiol 29: 781-785, 2008.

11. Roberts HC, Dillon WP and Smith WS: Dynamic CT perfusion to assess the effect of carotid revascularization in chronic cerebral ischemia. AJNR Am J Neuroradiol 21: 421-425, 2000.

12. Trojanowska A, Drop A, Jargiello T, Wojczal J and Szczerbo-Trojanowska M: Changes in cerebral hemodynamics after carotid stenting: evaluation with CT perfusion studies. J Neuroradiol 33: 169-174, 2006.

13. Barber PA, Demchuk AM, Zhang J and Buchan AM: Validity and reliability of a quantitative computed tomography score in predicting outcome of hyperacute stroke before thrombolytic therapy. ASPECTS Study Group. Alberta Stroke Programme Early CT Score. Lancet 355: 1670-1674, 2000.

14. Pexman JH, Barber PA, Hill MD, et al: Use of the Alberta Stroke Program Early CT Score (ASPECTS) for assessing CT scans in patients with acute stroke. AJNR Am J Neuroradiol 22: 1534-1542, 2001.

15. Mori T, Fukuoka M, Kazita K and Mori K: Follow-up study after intracranial percutaneous transluminal cerebral balloon angioplasty. AJNR Am J Neuroradiol 19: 1525-1533, 1998.

16. Feldman RL, Trigg L, Gaudier J and Galat J: Use of coronary Palmaz-Schatz stent in the percutaneous treatment of an intracranial carotid artery stenosis. Cathet Cardiovasc Diagn 38: 316-319, 1996.

17. Higashida RT, Furlan AJ, Roberts H, et al: Trial design and reporting standards for intra-arterial cerebral thrombolysis for acute ischemic stroke. Stroke 34: e109-e137, 2003.

18. Kalia J, Wolfe T and Zaidat OO: Limb-shaking transient ischemic attack masquerading as lumbar radiculopathy from pericallosal artery stenosis treated successfully with intracranial angioplasty and stenting. J Stroke Cerebrovasc Dis 19: 169-173, 2010. 